\title{
ASSOCIATION OF AFRICAN UNIVERSITIES (AAU) ASSOCIATION DES UNIVERSITES AFRICAINES (AUA)
}

P.O. Box 5744

Accra North (Ghana) 1967

Tel: (21) 663670

Fax: (21) 774338

Telex: 2284 adua gh

Cables: afuniv

President: Prof. Mahmoud Naguib Hosni Secretary-General: Prof. Donald E.U. Ekong

An international non-governmental organization which serves as the apex and principal forum for consultation, exchange of information, and cooperation among universities in Africa. Its programmes cover three core areas: Documentation and Information; Human Resources Development; and Strengthening Teaching and Research Capacities. AAU also works in close collaboration with international assistance organizations and national non-university institutions. Its activities include studies and publications, training workshops, seminars, conferences, and staff and student exchange.

Principal publications: $A A U$ Newsletter (three times a year); Directory of African
Universities (every two years); Reports of Conferences, Seminars, and Workshops.

L'Association des universités africaines (AUA) est une organisation internationale non-gouvernementale. Elle tient le rôle d'organisation centrale et de tribune principale pour la consultation, l'échange d' informations et la coopération parmi les universités africaines. Ses programmes s'intéressent à trois domaines essentiels: la documentation et l' information; le développement des ressources humaines et le renforcement de l' enseignement et de la recherche. L'AAU collabore aussi étroitement avec les associations d'aide internationale et avec les institutions nationales extra-universitaires. Ses activités comprennent: études et publications; ateliers de formation; séminaires; conférences et échanges de professeurs et d'étudiants.

Publications principales: "AAU Newsletter" (trois fois par an);"Directory of African Universities" (tous les deux ans); Rapports de conférences, séminaires et ateliers. 